Universidade Tecnológica Federal do Paraná - UTFPR

Campus Ponta Grossa - Paraná - Brasil

ISSN: 1981-3686 / v. 01, n. 02: p. 26 - 36
Revista Brasileira de Tecnologia
Agroindustrial

\title{
EQUIVALÊNCIA DE DULÇOR E PODER EDULCORANTE DE NÉCTARES DE GOIABA ADOÇADOS COM DIFERENTES EDULCORANTES
}

\section{EQUI-SWEETNESS AND SWEETENING POWER OF GUAVA NECTARS SWEETNED WITH DIFFERENT SWEETENING AGENTS}

\author{
Carlos Alexandre Koguishi de Brito ${ }^{1}$; Vitor Hugo Alves Câmara ${ }^{2}$; Helena Maria André Bolini ${ }^{3}$ \\ ${ }^{1}$ State University of Campinas - UNICAMP - Campinas - Brasil brito@fea.unicamp.br \\ ${ }^{2}$ State University of Campinas - UNICAMP - Campinas - Brasil vhalves@fea.unicamp.br \\ ${ }^{3}$ State University of Campinas - UNICAMP - Campinas - Brasil hellini@fea.unicamp.br
}

\begin{abstract}
Resumo
No presente estudo foi verificado a euivalência de doçura e no poder edulcorante de néctares de goiaba adoçados com diferentes agentes adoçantes. Foram avaliados: aspartame, sucralose, mistura ciclamato/sacarina 2:1, Stevia (Stevia rebaudiana (Bert.) Bertoni) e acessulfame-K, tendo como referência a sacarose. Primeiramente foi determinada a doçura ideal, utillizando-se escala do ideal com 30 provadores consumidores de néctar de goiaba. Em seguida, foi determinada a doçura equivalente à sacarose (na doçura considerada ideal) para cada edulcorante estudado, e seu poder edulcorante através do método de estimação de magnitude, utilizando-se uma equipe de 10 provadores selecionados e treinados. A doçura ideal de sacarose foi de 9,6\%
\end{abstract}

Palavras-chave: goiaba; edulcorantes; poder edulcorante.

\section{Introdução}

A tendência das indústrias que se preocupam em promover a saúde e prevenir doenças, está em investir no desenvolvimento de tecnologias para a produção de alimentos com baixas calorias e reduzido teor de gordura, mantendo suas qualidades nutricionais. Um dos estudo mais freqüentes é o uso de edulcorantes não calóricos que sejam semelhantes em função organoléptica à sacarose. (FERNANDES, 2001).

Por simples problemas de estética ou por problemas de saúde, o homem está substituindo o conhecido e consagrado açúcar (sacarose) por produtos conhecidos como edulcorantes, compostos com 
sabor semelhante a sacarose, porém, de baixo valor calórico ou completamente sem calorias (ANGELLUCI,1989).

A sacarina, o ciclamato, o aspartame, a sucralose, o acessulfame -K e o extrato de folhas de Stevia, todos edulcorantes permitidos pela Legislação Brasileira (BRASIL, 1995) para utilização em alimentos e bebidas dietéticas, possuem características sensoriais que podem diferir em função do produto em que se encontram. Por exemplo, um mesmo edulcorante que provoque a percepção de um gosto residual amargo intenso em um produto, pode ter esta característica atenuada em outro produto com outra estrutura química (CARDOSO, 2004).

A substituição da sacarose em formulações de alimentos é realizada através de estudos sensoriais que permitam o conhecimento prévio das concentrações dos adoçantes a serem utilizados e suas doçuras equivalentes em sacarose. O método mais aplicado é o de estimação da magnitude e representação gráfica dos resultados normalizados, através da Lei de Stevens ou "power function" (MOSKOWITZ , 1970; STONE \& OLIVER, 1969).

Tunaley et al. (1987) avaliaram a doçura de nove diferentes adoçantes, comparando-os a um padrão de glicose em solução a 5\%. Verificaram que houve muita variação nos dados obtidos, em relação aos provadores. Os autores concluíram que esta variação é dependente do tipo do adoçante, pois quanto mais complexa a percepção total, maior foi a variação nos resultados. E o aspartame foi o adoçante que apresentou características mais próximas da sacarose em doçura equivalente a uma solução de sacarose a 5\%. E Holmer(1988) observou que o mesmo adoçante apresentou gosto doce semelhante ao da sacarose e ausência de gosto residual em uma solução equivalente de sacarose a $10 \%$.

O princípio da estimação de magnitude, ou função de potência, proporciona a obtenção de diversas ferramentas importantes para a avaliação de alimentos através da análise sensorial. Os valores obtidos dos resultados dos provadores e os valores das concentrações avaliadas são normalizados, calculados os logaritmos desses resultados e colocados em um gráfico em coordenadas logarítmicas. Para cada adoçante (ou outro composto) é obtida uma reta, a qual obedece à lei de Stevens, ou "power function": $\mathrm{S}=\mathrm{aC}$, onde $\mathrm{S}$ é o estímulo percebido, C é a concentração do estímulo, a é antilog do valor de Y no intercepto, e n é o coeficiente angular da reta. Regiões das retas dos adoçantes em que estão em mesmo nível, paralelo ao eixo da abcissa, possuem poder edulcorante equivalentes (MOSKOWITZ, 1970).

A escala-do-ideal é o método afetivo mais aplicado para se medir a quantidade ideal de um determinado componente a ser adicionado no alimento ou bebida para provocar a melhor aceitação e preferência de um grupo de julgadores. Nesta análise, a equipe de provadores avalia as amostras e 
registra suas respostas em escala específica, o quão ideal estas amostras encontram-se, em relação ao atributo que se deseja avaliar (por exemplo doçura, textura e outros), conforme o método de Vickers (1988).

A goiaba (Psidium guajava L.) da família Myrtaceae é uma fruta originária do México, América Central e Norte da América do Sul. A fruta é saborosa e perfumada, cuja polpa colore-se do branco ao amarelo, rosa ou vermelho. Uma das frutas mais completas e equilibradas em relação ao valor nutritivo (SILVA, 1996).

O cultivo da goiaba é um dos mais importantes em países tropicais e subtropicais. O Brasil é um dos maiores produtores mundiais da fruta (FERREIRA, 2000).

O Estado de São Paulo é o maior produtor de goiaba no país, responsável por mais de 60\% do volume nacional da fruta e 55\% desse volume é destinado à industrialização. Além do consumo in natura, produtos industrializados como goiabada, geléia e néctar são a principal forma de consumo da fruta (AGRIANUAL, 2004).

O objetivo do presente estudo foi determinar a doçura ideal para néctar de goiaba, adoçado com sacarose, e determinar a quantidade equivalente de diferentes edulcorantes para promoverem a mesma doçura, e também determinar o poder edulcorante em relação à sacarose.

\section{Material e Métodos}

\subsection{Material}

Foi utilizado base de néctar de goiaba (comercial), preparado conforme as instruções do fabricante. O néctar assim preparado foi adoçado com cinco diferentes edulcorantes: aspartame puro (Nutrasweet); extrato de folhas de estévia (Steviafarma do Brasil); Mistura ciclamato / sacarina na proporção de 2:1; sucralose (Splenda, Johnson), sacarose (Sigma Chemical Co.) e acessulfame-K (Slim diet).

\subsection{Análise sensorial}

\subsubsection{Determinação da concentração ideal de sacarose}


A determinação da concentração ideal de sacarose (\%) a ser adicionada ao néctar de goiaba foi realizada através de um teste de aceitação, utilizando escala do ideal (LAWLESS \& HEYMANN, 1999).

Foram utilizados 30 voluntários consumidores de néctar de goiaba. As amostras foram apresentadas de forma monádica, sendo testadas as concentrações de 5,0; 7,5; 10,0; 12,5 e 15\% de sacarose.

\subsubsection{Pré-seleção da equipe de provadores}

A pré-seleção dos provadores foi realizada através de testes triangulares, aplicados à análise seqüencial (MEILGAARD et al, 1999). Para os testes, foram utilizadas duas amostras de néctar de goiaba, adoçadas com sacarose, que apresentavam diferença significativa entre si ao nível de 0,1\%. Para determinar a diferença de concentração entre estas duas amostras foi realizado um teste pareado com 30 provadores.

Na análise seqüencial foram utilizados os valores para p=0,45 (máxima inabilidade aceitável), p1=0,70 (mínima habilidade aceitável), e para os riscos $\alpha=0,05$ (probabilidade de aceitar um candidato sem acuidade sensorial) e $\beta=0,05$ (probabilidade de rejeitar um candidato com acuidade sensorial).

\subsubsection{Determinação da equivalência de doçura}

A medida do poder edulcorante relativo dos edulcorantes e da mistura de edulcorantes foi realizada de acordo com o método de estimação de magnitude (MEILGAARD et al, 1999), o qual proporciona a medida quantitativa direta da intensidade subjetiva de doçura.

As amostras foram apresentadas aos provadores através de blocos completos casualizados, acompanhadas de uma referência de néctar de goiaba adoçado com sacarose na concentração de 9,6\% (concentração ideal de sacarose a ser adicionada ao chá-mate, determinada no teste de aceitação com escala do ideal para sacarose).

Foram utilizados dez provadores selecionados em função do poder discriminativo através de análise seqüencial. Estes provadores foram treinados para utilizarem escalas de magnitude com padrões de intensidades de doçura diferentes. 
Para as avaliações de cada edulcorante em néctar de goiaba foram utilizadas as séries de concentrações apresentadas na Tabela 1, as concentrações centrais utilizadas, foram baseadas em dados da literatura e para o cálculo das demais concentrações foi utilizado o fator de multiplicação 1,6.

Tabela 1. Concentrações de sacarose, aspartame, estévia e sucralose, utilizadas para determinação da equivalência de doçura em relação à concentração de 9,6\% de sacarose em néctar de goiaba

\begin{tabular}{cccccc}
\hline Estímulo & \multicolumn{4}{c}{ Concentrações para determinação da equivalência de doçura à 9,6\% de sacarose* } \\
\hline Sacarose & 3,7500 & 6,0000 & 9,600 & 15,360 & 24,576 \\
Aspartame & 0,0215 & 0,0344 & 0,0550 & 0,0880 & 0,1408 \\
Ciclamato/sacarina & 0,0141 & 0,0225 & 0,0360 & 0,0576 & 0,0922 \\
Stévia & 0,0391 & 0,0625 & 0,1000 & 0,1600 & 0,2560 \\
Sucralose & 0,0063 & 0,0100 & 0,01600 & 0,0256 & 0,0410 \\
Acessulfame K & 0,0200 & 0,0340 & 0,0550 & 0,0880 & 0,1408 \\
\hline
\end{tabular}

* Concentrações em porcentagem (p/v) Fonte: CARDELLO, 1999; CARDOSO et al, 2004.

Neste método, os provadores selecionados e treinados receberam uma amostra referência, com intensidade designada por um valor arbitrário de doçura 100, seguida de várias amostras codificadas e balanceadas, com intensidades maiores ou menores que a referência (conforme indicado na Tabela 1). Solicitou-se aos provadores que estimassem as intensidades de doçura das amostras codificadas em relação à referência. Por exemplo, se a amostra apresentasse o dobro da doçura da referência, deveria receber valor 200, se apresentasse a metade da doçura, 50 e, assim por diante.

Para a análise dos dados, os valores de magnitude de doçura estimados foram convertidos para valores logarítmicos e expressos utilizando média geométrica. As curvas para concentração versus resposta sensorial, para cada edulcorante, foram correspondentes a uma função de potência ("power function") com a seguinte característica: $S=a C^{n}$, onde $\mathrm{S}$ é a sensação percebida, C é a concentração do estímulo, a é o antilog do valor de y no intercepto e n é o coeficiente angular da reta obtida (MOSKOWITZ , 1970).

\section{Resultados e Discussão}

A opinião dos provadores, verificada no teste utilizando a escala do ideal, foi transformada em dados numéricos $(-4,5$ a $+4,5)$, sendo que a opinião “ideal” correspondia ao valor 0. A partir da equação da reta obtida (Figura 1), calculou-se a concentração ideal de sacarose a ser adicionada ao néctar de goiaba que foi de 9,59\%. Para facilitar os experimentos futuros optou-se por utilizar 9,6\% como concentração ideal de sacarose 
O valor de $\mathrm{R}^{2}$ acima de 0,9 representa uma ótima correlação linear segundo Callegari-Jacques (2003). A partir da concentração de sacarose, para provocar a doçura ideal do néctar de goiaba, foi utilizada a metodologia estimação de magnitude.

Estão representados graficamente a relação entre a percepção sensorial de doçura e concentração para cada edulcorante (Figura 2) em coordenadas cartesianas com os valores calculados em logaritmo de base 10.

A partir dos resultados encontrados na Tabela 2, foi possível calcular a quantidade equivalente das substâncias adoçantes avaliadas para proporcionar a mesma doçura que a sacarose a 9,6\%, em néctar de goiaba. Tais quantidades encontram-se na Tabela 3.

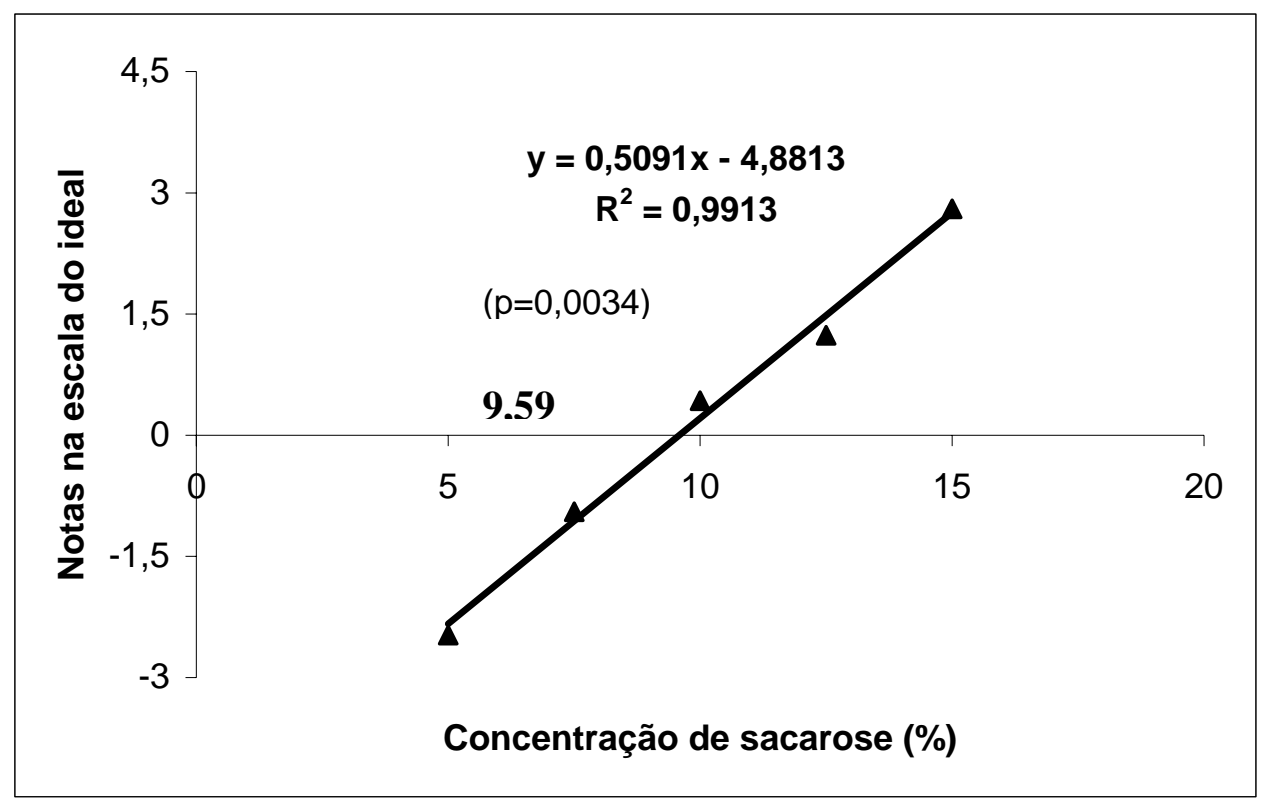

Figura 1. Gráfico e equação da reta no teste para a determinação da concentração ideal de sacarose a ser adicionada no suco de goiaba

Tabela 2. Coeficiente angular, intercepto na ordenada, coeficiente de correlação linear e função potência ("Power Function”) dos resultados obtidos nos testes utilizando escala de magnitude, para determinar as equivalências de doçura dos edulcorantes em relação à concentração de 9,6\% de sacarose em néctar de goiaba 


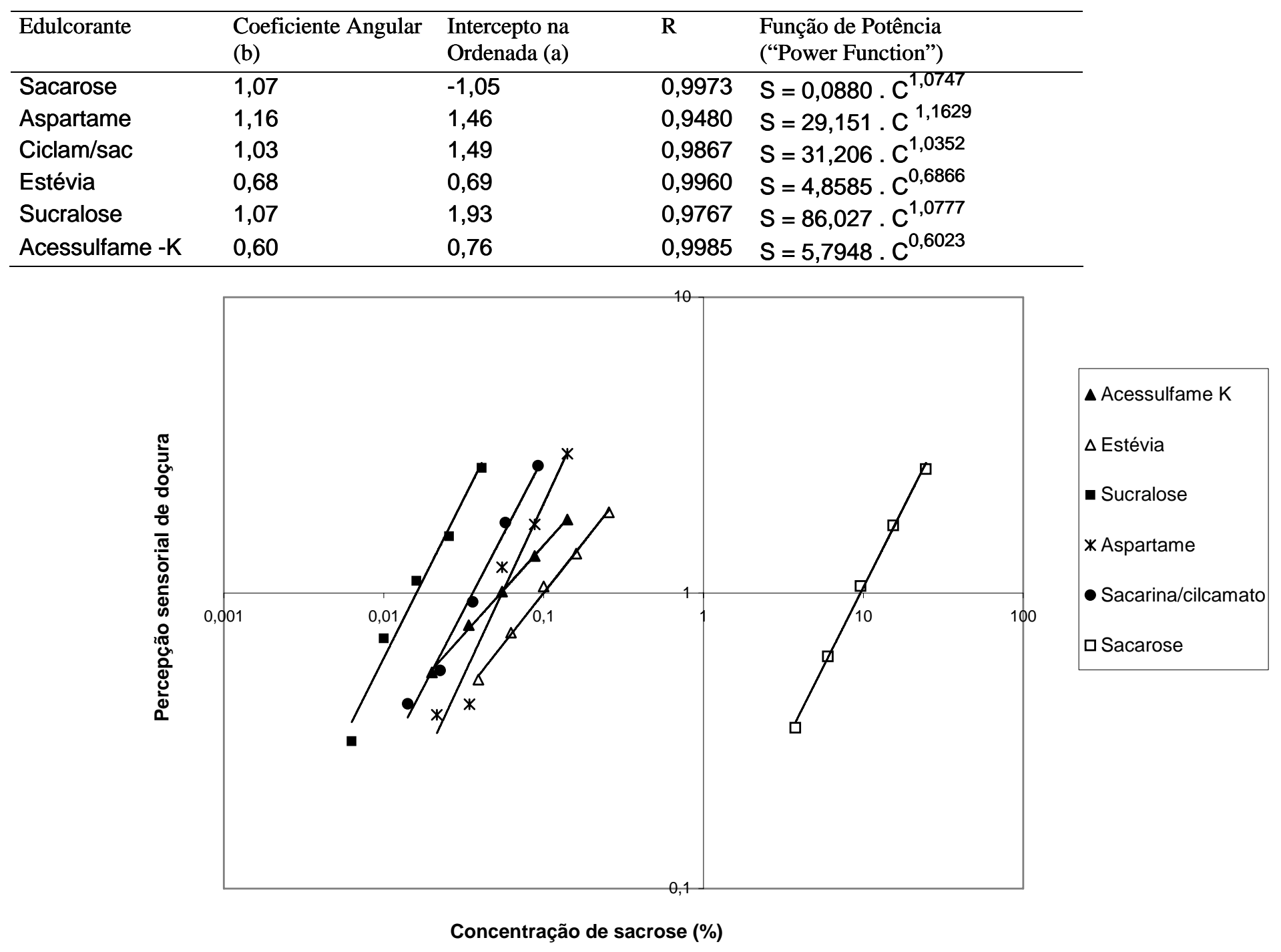

Figura 2. Relação entre percepção sensorial de doçura e concentração dos edulcorantes em relação à sacarose 9,6\% para néctar de goiaba

$\mathrm{R}=$ Coeficiente e $\mathrm{C}$

$\mathrm{S}=$ Estímulo perceb

$\mathrm{C}=$ Concentração d

Tabela 3. Concentração de aspartame, ciclamato/sacarina, estévia, sucralose e acessulfame- K que equivale à concentração de 9,6\% de sacarose em néctar de goiaba 


\begin{tabular}{cc}
\hline Edulcorante & $\begin{array}{c}\text { Concentração equivalente à 9,6 \% de } \\
\text { sacarose em néctar de goiaba }\end{array}$ \\
\hline Aspartame & 0,0550 \\
Ciclamato/sacarina & 0,0360 \\
Estévia & 0,1000 \\
Sucralose & 0,0160 \\
Acessulfame -K & 0,0541 \\
\hline
\end{tabular}

O resultado encontrado foi semelhante ao observado por Cardello et al (1999 a) e Cardoso et al (2004) para a determinação de equivalência de doçura na concentração de $10 \%$ em solução aquosa. Sugerindo que a matriz (néctar de goiaba) não interferiu na percepção de doçura dos edulcorantes

Apenas a amostra adoçada com estévia obteve concentração maior daquela permitida pela legislação brasileira que é de 0,060g/100g (BRASIL, 2001).

Com esses valores foi calculado o poder edulcorante das substâncias, as quais encontram-se na Tabela 4. O poder edulcorante é definido como o número de vezes que uma substância é mais doce que a sacarose.

Tabela 4. Poder edulcorante em relação à concentração de 9,6\% de sacarose em néctar de goiaba

\begin{tabular}{cc}
\hline Edulcorante & Poder edulcorante \\
\hline Aspartame & 175 \\
Ciclamato/sacarina & 267 \\
Estévia & 96 \\
Sucralose & 600 \\
Acessulfame -K & 177 \\
\hline
\end{tabular}

O menor valor de potência edulcorante foi observado para a estévia, o mesmo foi observado por Cardello et al (1999 b) que encontrou valor de 97 para a potência da estévia sem solução com 10\% de sacarose em água e Umbelino (2005) obteve valor de 94 para suco de manga equivalente à 7,5\% de sacarose.

Os valores de potência encontrados para aspartame e ciclamato foram semelhantes aos valores obtidos por Cardello et al (1999) em solução aquosa de 10 \% de sacarose 185 e 274 respectivamente. E o valor da sucralose foi idêntico ao citado por Jenner (1989) numa solução de sacarose à 5\%, poder adoçante 600 vezes mais doce que a sacarose.

Os edulcorantes aspartame e acessulfame-k apresentaram potência similar, o mesmo ocorreu com chá - mate gelado na concentração de 8,3\% de sacarose obtido por Cardoso et al (2004).

Esses resultados comprovam que a percepção dos gostos das substâncias depende de vários fatores, entre estes o meio de dispersão em que se encontram e da classe química. E quanto maior a similaridade do edulcorante com a doçura da sacarose e ausência de amargor melhor será a correlação 
nos resultados de determinação das concentrações de edulcorantes, permitindo uma aplicação em uma grande variedade de alimentos e bebidas.

\title{
4. Conclusão
}

A partir dos resultados obtidos foi possível concluir que:

- A doçura ideal de sacarose foi de 9,6\% para o néctar de goiaba;

- Não houve diferença de percepção de doçura da matriz (néctar de goiaba) para a concentração de 10\% em solução aquosa citada na literatura por Cardello et al (1999). Sugerindo que a matriz não interfere na percepção da doçura;

- Apenas a estévia ultrapassou os limites máximos da legislação brasileira nas concentrações equivalentes à sacarose 9,6\% e na determinação da escala do ideal das quantidades de edulcorantes a serem adicionadas no néctar de goiaba;

- O menor valor de poder edulcorante também foi observado para a estévia, provavelmente pelo acentuado gosto amargo;

- A potência edulcorante obedeceu a seguinte ordem crescente no néctar de goiaba à concentração de 9,6\% de sacarose: estévia, aspartame, acessulfame-K, ciclamato/sacarina e sucralose.

\begin{abstract}
In this work the effect of temperature of consumption in the equi-sweetness and sweetening power of guava nectars sweetened with different sweetening agents. The panelists had evaluated: aspartame, sucralose, mixture of cyclamate/saccharin (2:1), Stevia [Stevia rebaudiana (Bert.) Bertoni] and acesulfame-K as sweeteners, having sucrose as reference. Firstly the ideal sweetness was determined by just-about-right scale with 30 consumers of guava nectar. After the ideal sweetness of sucrose determination, the equi-sweetness (in the same ideal sweetness) for each sweetening agent was determined, and its sweetening power, for such was applied the method of magnitude evaluation, with a panel of 10 selected and trained panelists. The ideal sweetness of sucrose concentration was of $9.6 \%$ without significant difference ( $p \leq 0.05$ ).
\end{abstract}

Keywords: guava; sweeteners; sweetness potency.

\section{Referências}

AGRIANUAL, 2003: Anuário da agricultura brasileira. São Paulo: FNP Consultoria \& Comércio, 2004. p. 314-318.

ANGELUCCI, E. Edulcorantes e adoçantes em alimentos. Casa da Agricultura, v. 2, n. 11, p. 14-15, 1989. 
BRASIL. Agência Nacional de Vigilância Sanitária /Ministério da Saúde. Resolução n. 3 de 2 de Janeiro de 2001. Regulamento Técnico que aprova o uso de aditivos edulcorantes estabelecendo seus limites máximos para alimentos e bebidas dietéticas; Diário Oficial União, Brasília, 2001.

BRASIL. Ministério da Saúde. Secretaria de Vigilância Sanitária. Portaria no. 318 de 24 de novembro de 1995. Aprova o uso de Sucralose com a função de edulcorante em alimentos e bebidas dietéticas; Diário Oficial da República Federativa do Brasil, Brasília, no. 227, p.194061, 28 nov. 1995.

CARDELLO, H.M.A.B., DA SILVA, M.A.A.P., DAMÁSIO, M.H. Análise descritiva quantitativa de edulcorantes em doçura equivalente a sacarose em solução a 10 \% em pH neutro e ácido. In: ALMEIDA, T.C.A.; HOUGH, G.; DAMÁSIO, M.H.; DA SILVA, M.A.A.P. (ed). Avanços em Análise Sensorial. São Paulo: Livraria Varela. 1999 a. p.213-226

CARDELLO, H.M.A.B., DA SILVA, M.A.A.P., DAMÁSIO, M.H. Measurement of the relative sweetness of stevia extract, aspartame and cyclamate/saccharin blend as compared to sucrose at differents concentrations. Plant Foods for Human Nutrition, v.54 n.2, p. 119-130, 1999b.

CARDOSO, J.M.P., BATTOCHIO, J. R., CARDELLO, H. M. A. B. Equi-sweetness and sweetening power of different sweetening agents in differents temperatures of consumption of tea drink in soluble power. Ciência e Tecnologia de Alimentos, vol.24, no.3, p 448-452, 2004.

FERNANDES, L. M.; PEREIRA, N. C.; MENDES, E. S.; LIMA, O. C. M.L. \& COSTA, S. C. Clarificação do extrato aquoso de Stevia rebaudiana (Bert.) Bertoni utilizando o cacto, Cerus peruvianis. Acta Scientiarium, Maringá, v.23, n. 6, p. 1369-1374, 2001.

FERREIRA, P.R. Goiaba deve atrair mais consumidores. Gazeta Mercantil, P. B-20, 25 de Abril, 2000.

JENNER, M.R. Unveiling its properties and applications. In: GREMBY, T.H. Progress in sweeteners. New York: Elsevier Applied Science, 1989. p. 121-142.

LAWLESS, H.T.; HEYMANN, H. Sensory evaluation of food: principles and practices. Gaithersburg: Aspen Publishers, 1999. 827 p.

MEILGAARD, M., CIVILLE, G.V., CARR, B.T. Sensory evaluation techniques. $3^{\text {rd }}$. ed., New York: CRC Press, 1999, 387p.

MOSKOWITZ, H.R. Ratio scales of sugar sweetness. Perception Psychophys, v.7, p.315-20, 1970.

SILVA, S.P. Frutas no Brasil. São Paulo: Empresa das Artes; 1996. 232p.

STONE, H., OLIVER, S.M. Measurement of the relative sweetness of selected sweeteners and sweetener mixtures. Journal of Food Science, v.34, p.215-22, 1969.

TUNALEY, A., THOMSON, D.M.H., McEWAN, J.A. Determination of equi-sweet concentrations of nine sweeteners using a relative rating technique. International Journal of Food Science and Technology, v. 22, p. 627-351 1987.

UMBELINO, D.C. Caracterização sensorial por análise descritiva quantitativa e análise tempo-intensidade de suco e polpa de manga (Magnífera indica L.) adoçados com diferentes edulcorantes. Tese de Doutorado. Universidade Estadual de Campinas. Faculdade de Engenharia de Alimentos. Campinas-SP. 2005. 190 p.

VICKERS, Z. Sensory specific satiety in lemonade using a just right scale for sweetness. Journal of Sensory Study, V.3, n1, p.1-8, 1988.

Nome Completo: Carlos Alexandre Koguishi de Brito; Vitor Hugo Alves Câmara; *Helena Maria André Bolini

Filiação Institucional: Faculdade de Engenharia de Alimentos - Universidade Estadual de Campinas UNICAMP 
Departamento: Alimentos e Nutrição (Laboratório de Análise Sensorial)

Função ou cargo ocupado: Aluno de pós graduação, graduação e *docente

Endereço para correspondência: Rua Monteiro Lobato, 80 Cidade Universtiária "Zeferino Vaz Dias" Barão Geraldo Campinas -SP C.P. 6121 CEP: 13083-862.

Telefone para contato: *(19) 3521-4083

e-mail: brito@fea.unicamp.br; 